\title{
Questes
}

\section{Entre Bourguignons et Armagnacs, communauté urbaine et fidélités politiques (Troyes, 1429-1433)}

\section{Cléo Rager}

\section{(2) OpenEdition}

\section{Journals}

\section{Édition électronique}

URL : http://journals.openedition.org/questes/4337

DOI : 10.4000/questes.4337

ISSN : 2109-9472

\section{Éditeur}

Les Amis de Questes

\section{Édition imprimée}

Date de publication : 6 mai 2016

Pagination : 123-138

ISSN : 2102-7188

\section{Référence électronique}

Cléo Rager, «Entre Bourguignons et Armagnacs, communauté urbaine et fidélités politiques (Troyes, 1429-1433) », Questes [En ligne], 32 | 2016, mis en ligne le 10 mai 2016, consulté le 01 mai 2019. URL : http://journals.openedition.org/questes/4337 ; DOI : 10.4000/questes.4337 


\title{
Entre Bourguignons et Armagnacs, communauté urbaine et fidélités politiques (Troyes, 1429-1433)
}

\author{
Cléo RAGER \\ Université Paris 1 Panthéon-Sorbonne \\ Institut de Recherche et d'Histoire des Textes
}

En l'an mil CCCCXXIX apres les sieges de Montargis et Orleans, mis et tenuz par les anciens ennemis de nostre royaume les Angloys [...] le roy Charles [...] se mist sur les champs en armes pour venir a son sacre et couronnement reduire, recouvrer et mettre en son obeissance les villes, citez et pays detenuz et occupez par sesdits anciens ennemys et adversaires, et [...], en venant depuis la riviere de Loyre [...] il ne trouva ville ne cité qui a luy le voulsist reduire, incontinant qu'il fut arrivé devant nostre dicte ville de Troyes et que les habitans d'icelle dont plusieurs en y a encores en vie, eurent de luy congnoissance, le receurent comme leur vray, naturel et souverain seigneur et comme tel et les premiers sans quelconque contraincte, resistance ou difficulté lui feirent planiere, entiere et vraye obeissance, en mettant en la main et puissance nostre dicte ville comme ses bons, vrays et loyaulx subgectz ${ }^{1}$.

L'insistance sur la communauté urbaine unie autour de la fidélité royale retrouvée fait partie des lieux communs du discours troyen destiné au roi à la fin du $X V^{e}$ siècle. De cette loyauté précoce découle un certain

\footnotetext{
${ }^{1}$ Troyes, AM, fonds Delion, layette 1 , pièce 1 , cartulaire de la ville, $\mathrm{f}^{\mathrm{o}} 108$ : lettre de Charles VIII concernant l'exemption des habitants de Troyes, 18 mai 1486.
} 
nombre d'avantages et de privilèges accordés à la ville. Le 9 juillet 1429 , le futur Charles VII prend en effet le contrôle de la ville de Troyes, alors même que les villes voisines restent majoritairement sous fidélité bourguignonne. L'événement est d'importance pour le dauphin : c'est le chemin vers Reims et vers le sacre qui s'ouvre. Symboliquement, Troyes est aussi le lieu où «la majesté de Charles VII a été détruite le 21 mai $1420 »$, lors du «honteux traité de Troyes ${ }^{2} »$. Jusqu'au milieu du $\mathrm{XX}^{\mathrm{e}}$ siècle, l'interprétation de cet épisode de la guerre de Cent Ans par l'historiographie reste la même : celle d'une occupation par l'ennemi au cours de laquelle les habitants auraient été contre leur gré soumis au pouvoir bourguignon pendant une douzaine d'années, le mois de juillet 1429 étant celui d'une libération. Selon cette hypothèse, l'appartenance des habitants à une communauté politique n'est pas mise en doute ${ }^{3}$. Pourtant, à en croire les chroniqueurs, si, le $1^{\text {er }}$ août 1417, l'entrée des Bourguignons se fait dans l'enthousiasme de la foule, une décennie plus tard, lors de l'entrée du dauphin, l'assistance semble se réduire à quelques individus ${ }^{4}$.

\footnotetext{
${ }^{2}$ Colette Beaune, « Rois de France, rois de la fève », dans Royautés imaginaires (XII ${ }^{e}$ $X V I^{e}$ siècles). Actes du colloque organisé par le Centre de recherche d'histoire sociale et culturelle (CHSCO) de l'Université de Paris X-Nanterre (26 et 27 septembre 2003), dir. Colette Beaune et Henri Bresc, Turnhout, Brepols, 2005, p. 75-88, cit. p. 83.

${ }^{3}$ Comme l'écrit Théophile Boutiot à propos de Troyes : «Le chapitre que nous achevons a montré les habitants de Troyes attachés à leur roi. Ils ne subirent dans leurs murs le pouvoir étranger que parce que la ruse et la fourberie les avaient placés sous le joug des ennemis de la France et de leur naturel et souverain seigneur. [...] Aussitôt que les circonstances le permirent, les Troyens répudièrent une autorité que la contrainte seule avait pu leur faire subir », dans Théophile Boutiot, Guerre des Anglais, 1429-1435. Un chapitre de l'histoire de Troyes, Paris, Techener, 1861, p. 64. Il s'agit d'un extrait de Théophile Boutiot, Histoire de la ville de Troyes et de la Champagne méridionale, Troyes, Dufey-Robert, 1870, 5 vol.

${ }^{4}$ Ceci est bien décrit par Benoît Léthenet dans sa thèse de doctorat, «Comme l'on se doit gouverner ». La Guerre, la ville et le pouvoir. Mâcon (vers 1382-vers 1435), Thèse de doctorat soutenue sous la direction de Georges Bischoff, Université Marc Bloch, Strasbourg, 2012, p. 666 et suiv.
} 
Comment comprendre et évaluer les conséquences de ce changement politique pour la communauté urbaine? Quel rôle joue le facteur politique dans la redéfinition de cette dernière et de ses fidélités ?

La ville de Troyes conserve dans ses archives un document particulièrement riche d'instructions pour cette période, un registre de délibérations de la ville, contenant des procès-verbaux datés du mois de septembre 1429 (deux mois après la reddition de la ville au roi) à septembre $1433^{5}$. Il s'agit du premier registre de délibérations conservé par la ville, et le seul avant 1483, date de confirmation de la création de l'échevinage de Troyes. Connu des historiens et ayant fait l'objet d'une édition incomplète ${ }^{6}$, il a servi de source d'informations aux deux principaux historiens de la ville, Théophile Boutiot ${ }^{7}$ et Françoise Bibolet ${ }^{8}$. Cependant, qu'il s'agisse de sa forme ou de son fond, il suscite encore de nombreuses interrogations, dans le cadre de l'attention renouvelée de l'historiographie aux actes de la pratique'.

\footnotetext{
${ }^{5}$ Troyes, AM, fonds Boutiot, série A, registre 1.

${ }^{6}$ Alphonse Roserot, Le Plus Ancien Registre des délibérations du conseil de la ville de Troyes, Troyes, Librairie L. Lacroix, 1886.

${ }^{7}$ Théophile Boutiot, Guerre des Anglais, 1429-1435, op. cit.

${ }^{8}$ Voir entre autres Françoise Bibolet, «Le rôle de la guerre de Cent ans dans le développement des libertés municipales de Troyes », Mémoires de la Société d'agriculture, sciences et arts du département de l'Aube, Troyes, 1939, vol. 99, p. 295-315. Plus général, Françoise Bibolet, Chantal Rouquet, André Boisseau et Emmanuel Saint-Mars, Histoire de Troyes, Troyes, Les Éditions de la Maison du boulanger, 1997. Deux mémoires ont été menés à partir de ce registre, mais qui n'en étudient pas les caractéristiques codicologiques : Olivier Derouin, L'Information et son fonctionnement à travers les registres de délibération du conseil de ville de Troyes au $X V^{e}$ siècle, Mémoire de Maîtrise soutenu sous la direction de Claude Gauvard, Université de Reims, 1991 ; Sébastien Mérat, Troyes pendant la guerre de Cent Ans d'après les archives du Conseil de ville (1429-1433), Mémoire de Maîtrise soutenu sous la direction de Charles Vulliez, Université de Reims, 2001. Ce dernier mémoire a été synthétisé en un article: Sébastien Mérat, «Une ville dans la tourmente. Troyes pendant la guerre de Cent ans, d'après les archives du Conseil de ville, 1429-1433 », La Vie en Champagne, $\mathrm{n}^{\circ} 33$ (mars 2003), p. 28-32.

${ }^{9}$ Selon les méthodes de la «nouvelle érudition», tentant de «saisir l'écrit dans sa dimension et dans sa fonction pratique » : Étienne Anheim et Pierre Chastang, «Les
} 


\section{Une unité apparente}

Les deux chartes accordées par le roi à la ville le jour même de son entrée à Troyes, le 9 juillet 1429, sont encore conservées aux archives municipales : une lettre d'abolition par laquelle le roi accorde son pardon à la ville et une charte définissant plus précisément les conditions de ce $\operatorname{pardon}^{10}$. Le roi, dès sa première entrée entend ressouder la communauté urbaine autour de sa personne, par l'usage du serment ${ }^{11}$. Rappelons que celui-ci est à la base de l'autonomie politique des villes, de leurs libertés, et plus généralement, pour reprendre Bernard Guenée, le «fondement de l'ordre social ${ }^{12} »$. C'est un acte crucial du processus de paix.

Et premierement. Que leurs corps et biens, et un chacun d'eux, en quelque part qu'ils soient, donnez et non donnez, seront saufs a eux, en faisant serment de nous estre doresnavant et a tousjours bon et loyaux subjects ${ }^{13}$.

La forme du serment de fidélité classique de tout contrat féodal, le fidelitatis juramentum, et les circonstances dans lesquelles il s'inscrit,

pratiques de l'écrit dans les sociétés médiévales ( $\mathrm{VI}^{\mathrm{e}}-\mathrm{XIII}{ }^{\mathrm{e}}$ siècle) », Médiévales. Langues, textes, histoire, 2009, $\mathrm{n}^{\mathrm{O}} 56, \mathrm{p} .4$.

10 Troyes, AM, fonds Delion, layette 2, liasse 7, pièces 1 et 2 . Sur ce type de document, voir Claude Gauvard, «Pardonner et oublier après la guerre de Cent Ans. Le rôle des lettres d'abolition de la chancellerie royale française », dans Vergeben und Vergessen? Pardonner et oublier?, dir. Reiner Marcowitz et Werner Paravicini, Munich, R. Oldenbourg, 2009, vol. 94, p. 27-55.

11 Sur l'importance du serment dans les villes au bas Moyen Âge, voir les contributions au $\mathrm{n}^{\mathrm{O}} 39$ de la revue Histoire urbaine parue en 2014 et consacrée à ce thème.

${ }^{12}$ Bernard Guenée, «Non Perjurabis. Serment et parjure en France sous Charles VI », Journal des savants, 1989, vol. 3, n ${ }^{\circ}$ 1, p. 241-257. Voir aussi Nicolas Offenstadt, «Guerre civile et espace public à la fin du Moyen Âge. La lutte des Armagnacs et des Bourguignons », dans La Politisation. Conflits et construction du politique depuis le Moyen Âge, dir. Laurent Bourquin et Philippe Hamon, Rennes, PUR, 2010, p. 111129.

${ }^{13}$ Nicolas Camusat, Meslanges historiques ou recueil de plusieurs actes, traictez, lettres missives, et autres mémoires qui peuvent servir en la déduction de l'histoire, depuis l'an 1390 jusques à l'an 1580. Est adjouté un ancien formulaire pour les secrétaires du Roy, maison et Couronne de France avec les chartres expédiées en faveur de leur Collège, Troyes, N. Moreau, 1619, p. 214-216 (nous soulignons). 
l'arrivée de Charles VII dans les villes reconquises, n'ont rien d'exceptionnelles. Le second article précise les conditions de ce serment.

Item, que si aucun des manans et habitans de la ville ou autres estans en icelle, ne veulent demeurer ne faire ledict serment, ils auront delay de vuider, et eulx departir d'icelle ville jusques a huict jours, et auront sauf-conduit pour aller ou bon leur semblera, avecques temps et induce de deux mois pour transporter leurs biens meubles, et faire leur profit et aliénation de leurs héritages et biens immeubles.

Rester en ville suppose donc de prêter serment au roi, et la communauté urbaine se comprend alors d'abord dans son union autour de l'hommage rendu à un seigneur commun.

Des procédures d'enquêtes contre les traîtres sont mises en place par les officiers royaux pour renforcer la communauté. L'exclusion des personnes infidèles au roi est contenue dans le serment même fait à celuici. Ainsi, le 13 mars 1430, le bailli regrette « qu'il y en avoit plusieurs en ceste ville qui estoient affectez au party contraire » et déclare « que se il en y avoit encores, qui se voulsissent departir de ceste ville, que sceurement s'en allassent ${ }^{14}$ ». La ville entreprend des mesures d'enquête sur les habitants et encourage les délations. Les décisions peuvent être radicales, comme le 19 avril 1430 :

deliberé que la maisonnette estans aupres la porte du Beffroy, en laquelle demeure Moslé, soit démolie, et ledit Moslé bouté hors d'icelle, pour aucuns rapports faiz de sa personne qui sont mauvaiz ${ }^{15}$.

Si cela apparaissait déjà en filigrane dans les mesures d'exclusion mises en place par la ville, il est aussi dit clairement dans le registre que l'appartenance à la communauté urbaine recoupe l'appartenance au parti

\footnotetext{
${ }^{14}$ Troyes, AM, Fonds Boutiot, série A, registre $1, \mathrm{f}^{\mathrm{o}} 18 \mathrm{r}^{\mathrm{o}}$.

15 Troyes, AM, Fonds Boutiot, série A, registre $1, \mathrm{f}^{\mathrm{o}} 19 \mathrm{r}^{\circ}$.
} 
du roi, comme le 27 janvier 1430 : «Que homme tenant le party contraire du Roy nostre sire, pour quelconque saufconduit qu'il ait, ne soit mis dedens la ville ${ }^{16} »$.

Cette définition de la communauté passe aussi par l'adoption de signes de reconnaissance communs à tous, allant dans le sens d'une communauté politique visible. L'appartenance urbaine et royale est concrétisée par le port d'une croix droite, sans doute blanche, car la croix blanche est le symbole du parti du roi ${ }^{17}$. Ainsi, le $1^{\text {er }}$ août 1431 , il est « ordonné que doresenavant chacun porte la croix droitte cousue en sa robe et que chacun ait l'euil a son compaignon en rapportant a justice la faulte qu'il y congnoistra ${ }^{18} »$. La ville de Troyes n'échappe pas à l'accroissement général des signes visuels et politiques dans ce temps de guerre civile ${ }^{19}$. Cette appartenance vient aussi marquer l'espace. Le 4 juillet 1433, le duc de Bourgogne approche, il a passé la nuit dans un bourg proche de Troyes. On décide alors que «soient faictes quatre banieres armoyees des armes du roy nostre sire qui seront mises sur les portes de ceste $\operatorname{cité}^{20} »$. L'espace urbain adopte les armes de son seigneur.

16 Troyes, AM, Fonds Boutiot, série A, registre $1, \mathrm{f}^{\mathrm{0}} 23 \mathrm{r}^{\mathrm{o}}:$ «...mais soit par les pardessus mis a autre chemin par dehors; et se ilz avoient a besongner en la ville, que on les face logier dehors et parler a ceulx a qui ilz auront a besongner ». Ou encore $\mathrm{f} 23 \mathrm{v}^{\circ}$ : «Item, a esté deliberé que homme souppeconné tenir party contraire, ne qui soit retrait en frais non obeïssant au roy, ne soit rappellé ne receu en ceste ville, pour quelconque requeste qui en soit faicte. »

${ }^{17}$ Sur la «guerre des signes », voir Simona Slanička, Krieg der Zeichen. Die visuelle Politik Johanns ohne Furcht und der armagnakisch-burgundische Bürgerkrieg, Göttingen, Vandenhoeck \& Ruprecht, 2002. Sur l'usage de signes distinctifs par les Armagnacs et Bourguignons, voir Nicolas Offenstadt, « Armagnacs et Bourguignons. L'affreuse discorde », dans La Guerre de Cent ans. L'Angleterre et la France en guerre, 1300-1450, dir. Christopher Allmand, Paris, Payot, 2013, p. 120.

18 Troyes, AM, Fonds Boutiot, série A, registre $1, \mathrm{f}^{\mathrm{O}} 54 \mathrm{v}^{\mathrm{o}}$, ou encore le 15 septembre $1433\left(\mathrm{f}^{\mathrm{O}} 158 \mathrm{v}^{\circ}\right)$ : «a esté ordonné que chacun porte la croix droitte pour ensaigne $»$.

${ }^{19}$ Simona Slanička, Krieg der Zeichen, op. cit., cité par Benoît Léthenet, Comme l'on se doit gouverner, op. cit., p. 118. Voir aussi p. 119 sur la multiplication des signes du côté bourguignon, comme à Châlons-en-Champagne.

${ }^{20}$ Troyes, AM, série $A$, registre $1, \mathrm{f}^{\mathrm{o}} 152 \mathrm{v}^{\mathrm{o}}$. 
Plus encore, le registre lui-même évoque l'unité et la fidélité urbaine à la politique royale. Les références au roi y sont omniprésentes, Le bien de la ville et celui du roi sont confondus, comme est absente la distinction nette entre les statuts de citadin et de sujet du roi. Tout d'abord, la figure du roi est partout dans le registre. $33 \%$ des procèsverbaux mentionnent le roi $^{21}$, et particulièrement dans les deux premiers cahiers, composés sous l'autorité du bailli. Cela paraît cohérent car le rôle des officiers royaux est de représenter le roi et de rapporter ses paroles ${ }^{22}$. En outre, la fidélité au roi est affirmée clairement par le conseil municipal. Pour nous en convaincre, reportons-nous à la demande du bailli de Troyes effectuée le 13 mars 1430 alors qu'il revient d'un voyage auprès du roi :

messieurs ont respondu qu'ilz estoient tous au roy, pres d'obeïr a ses commendemens, et que en gardent leurs loiaultez envers lui ils garderoient tres bien ceste cité, et, a l'aide de Dieu, lui en rendront bon compte ${ }^{23}$.

Enfin, il semble que le bien de la communauté et le bien du roi aillent souvent de pair dans le registre. Les intérêts du roi et de la ville sont juxtaposés dans les délibérations, les mesures étant prises «pour le bien du roy nostre sire et de ladite ville ${ }^{24} \gg$. Les sizainiers, chargés de

\footnotetext{
${ }^{21}$ Contre par exemple, dans un contexte différent, $18 \%$ dans le premier registre de délibérations de Brignoles (1387-1391), chiffre considéré pourtant comme particulièrement élevé par Lynn Gaudreault, dans Lynn Gaudreault, Pouvoir, mémoire et identité. Le premier registre de délibérations communales de Brignoles (1387-1391), Montpellier, Presses Universitaires de la Méditerranée, 2014, p. 169.

${ }^{22}$ Troyes, AM, fonds Boutiot, série A, registre $1, \mathrm{f}^{0} 8-8 \mathrm{v}^{\circ}$. ou encore $\mathrm{f}^{0} 18$ : le bailli rapporte les paroles du roi: «en presence desquelz monseigneur le bailli, qui nouvellement estoit venu devers le roy, dist et exposa que il desplaisoit au roy des charges et oppressions que les ennemis faisoient porter et soustenir a ses subgez demourans en ceste ville...»

${ }^{23}$ Troyes, AM, fonds Boutiot, série $A$, registre $1, \mathrm{f}^{\mathrm{o}} 18 \mathrm{v}^{\circ}$, ou encore $\mathrm{f}^{\circ} 95 \mathrm{r}^{\mathrm{o}}$, le 14 juillet 1432: "Messieurs ont tous tres bon vouloir d'eulx emploier a l'aide et secours du roy ».

24 Troyes, AM, fonds Boutiot, série A, registre 1, $\mathrm{f}^{0} 42$, le 5 juin 1431. Lynn Gaudreault fait cette même observation pour le registre de Brignoles où les deux
} 
faire le guet, doivent prêter serment de « garder le bien et honneur du roy nostre sire et les clefz de la ville, senz y faire faulte ${ }^{25} »$. Les cris prononcés sur ordre du conseil sont placés sous l'autorité royale ${ }^{26}$. De même les citadins sont assimilés aux «subgez du roy », cette formule revenant à de multiples reprises ${ }^{27}$.

Mais si toutes ces mesures doivent être prises, c'est peut-être parce que la fidélité au futur Charles VII est loin d'être acquise dans la population. D'où la nécessité pour le parti delphinal et les officiers royaux de mettre en place une véritable politique de communication à destination de la communauté, dont le registre porte le témoignage.

\section{Officiers royaux et communication politique}

Les premiers mois, les assemblées et certainement toutes les décisions municipales dépendent des officiers royaux, qui ont la mission de mener au mieux la transition entre les fidélités bourguignonne et delphinale. Dans le premier cahier du registre, ces derniers ne sont jamais accompagnés de gens élus mais plutôt de notables qui semblent davantage suivre l'officier dans ses décisions que réellement intervenir, et qui forment le «conseil » de la ville. À partir du deuxième cahier, on lit

termes de l'honneur du roi et de l'utilité de la ville sont liés à maintes reprises, Lynn Gaudreault, Pouvoir, mémoire et identité, op. cit., p. 170.

${ }^{25}$ Troyes, AM, fonds Boutiot, série A, registre $1, \mathrm{f}^{\mathrm{O}} 6 \mathrm{v}^{\mathrm{o}}$, le 10 octobre 1429 : après la liste des sizainiers chargés de faire le guet, «tous lesquelz dessus nommez, sur ce ayans jour, a ma requeste, par devers monseigneur le bailli ont fait serrement de bien [et] loialement garder et faire garder par leurs compaignons, a leur tour, aux portes et autrepart, chascun endroit soy et a son tour, garder le bien et honneur du Roy nostre sire et les clefz de la ville, senz y faire faulte » (nous soulignons).

${ }^{26}$ Troyes, AM, fonds Boutiot, série A, registre $1, \mathrm{f}^{\mathrm{o}} 29-29 \mathrm{v}^{\mathrm{o}}$, le $1^{\mathrm{er}}$ mars 1431 : « Or escoutez, de par le roy nostre sire et du commendement de mon seigneur le bailli... ».

${ }^{27}$ Par exemple Troyes, AM, fonds Boutiot, série A, registre 1, f 21,13 mai 1430 : «Item des pilleries, appatissements, entreprinses et roberies que font les gens de guerre souffrir aux povres subgez du roy, messeigneurs ont primé à mon seigneur le bailli que pour le bien de justice, qui est le bien du Roy et de sa seignorie, il y pourvoye $[. .$.$] ». Aussi au \mathrm{f}^{0} 27$, le 7 février 1431 : «les povres subgez du roy » sont volés par les larrons. 
que «messires du conseil de Troyes » sont présents aux délibérations. Dès le troisième cahier et la date du 3 octobre 1431, on a des élus « avec auctorité de justice ». Cette mention est alors très souvent ajoutée à la présentation de la séance. Ce n'est qu'à partir du $1^{\mathrm{er}}$ octobre 1432 que toutes les délibérations sont faites «par messires les esleuz de la ville de Troies », pour les « affaires et choses communes de ladicte ville».

Si cette présence importante des officiers royaux dans le registre des délibérations municipales de la ville peut être retrouvée dans d'autres contextes urbains $^{28}$, l'évolution au cours d'un même registre est, semblet-il, une spécificité troyenne. En outre, les officiers semblent avoir un réel rôle municipal, et encadrer véritablement la mise en place d'une organisation politique dans la ville ${ }^{29}$. Dans la seule délibération antérieure au registre, conservée dans les archives de la ville, sous la forme de minute, l'assemblée est aussi tenue (sans précisions sur la nature juridique de cette présence) par Pierre le Tartrier, lieutenant du bailli,

${ }^{28}$ Katia Weidenfeld, Les Origines médiévales du contentieux administratif, XIV ${ }^{e}$ $X V^{e}$ siècles, Paris, De Boccard, 2001, p. 42. La présence d'officiers royaux lors des conseils de ville fait même l'objet d'une législation spécifique par Charles VI à Toulouse en 1389. Albert Rigaudière, «Règlementation urbaine et "législation d'État" dans les villes du Midi français aux XIII ${ }^{\mathrm{e}}$ et XIV ${ }^{\mathrm{e}}$ siècles », dans Gouverner la ville au Moyen Âge, dir. Albert Rigaudière, Paris, Anthropos, 1993, p. 141. Les exemples des registres de Sisteron (Alexandra Gallo, La Communauté de Sisteron (XIII ${ }^{e}-X I V^{e}$ siècles). L'exercice du pouvoir urbain, rythmes et enjeux, Thèse de doctorat soutenue sous la direction de Jean-Paul Boyer, Université d'Aix-Marseille I, Aix-enProvence, 2009, p. 200), de Brignoles (voir Lynn Gaudreault, Pouvoir, mémoire et identité, op. cit., p. 171-178 qui conclut ainsi son étude du bayle brignolais en le qualifiant de «trait d'union entre deux mondes, chevauchant la frontière floue et mouvante qui sépare le pouvoir urbain du pouvoir royal ») ou de Reims (Julien Briand, L'Information à Reims aux XIV et XV ${ }^{e}$ siècles, Thèse de doctorat soutenue sous la direction de Claude Gauvard, Université Paris 1 Panthéon-Sorbonne, Paris, 2012, tableaux p. 352) ont ce point commun avec celui de Troyes.

${ }^{29}$ Loïc Cazaux a bien vu le dialogue constant entre les élus et le bailli et souligne la place de celui-ci dans les délibérations, Loïc Cazaux, Guerre et Pouvoir. Les capitaines face à la justice dans le royaume de France au $X V^{e}$ siècle, Thèse de doctorat soutenue sous la direction de Claude Gauvard, Université Paris 1 PanthéonSorbonne, Paris, 2012, p. 52. 
accompagné toutefois par «messires les esleuz a $\operatorname{Troyes}^{30}$ ». Déjà au temps de la domination bourguignonne, les assemblées étaient placées sous surveillance, notamment par un capitaine en 1417-1418 ${ }^{31}$. Restent alors à préciser les modalités de cette relation du bailli au conseil et aux assemblées des habitants telles qu'elles apparaissent dans le registre: domination d'un acteur royal reprenant une ville récemment infidèle ou coopération avec une autorité municipale en pleine reconstruction ${ }^{32}$ ?

Les officiers royaux utilisent plusieurs moyens pour promouvoir le soutien au roi à travers la ville. Soulignons tout d'abord le rôle de la prédication et des ecclésiastiques, déjà remarqué en d'autres villes ${ }^{33}$. Apparaissent dans le registre les efforts déployés par la ville pour retenir sur place un prêcheur particulièrement efficace, frère Léonard Breton. Ses prédications sont d'autant plus encouragées par le conseil de ville qu'elles prônent la nécessité d'aimer le roi. Ainsi, le 29 janvier 1433,

et qui plus est, avoit le fait du roy nostre sire et de seignorie en toutes ses predicacions moult notablement recommandé, et exhorté le peuple a le aimer et soy tenir en sa bonne et vraye obeïssance; et pour ce tous les dessus ditz furent et ont esté d'un commun accord qu'il demeure, senz soy departir hors de ceste ville, et qu'ilz aimeroient mieulx que frere Didier et frere Estienne et dix autres de leur religion

\footnotetext{
${ }^{30}$ Troyes, AM, fonds Boutiot, AA7, liasse 3, pièce 2.

${ }^{31}$ Gustave Dupont-Ferrier, Gallia regia ou État des officiers royaux des bailliages et des sénéchaussées de 1328 à 1515, Paris, Impr. nationale, 1961, vol. VI, p. 95, $\mathrm{n}^{\mathrm{o}} 22735$.

${ }^{32}$ C'est avant tout sur le mode de l'entraide et de la coopération pour parer aux difficultés de la guerre que Françoise Bibolet envisage ce rapport, sans commenter l'évolution de la présence des officiers royaux qui apparaît dans les sources à ce moment. Françoise Bibolet, «Les fonctionnaires royaux à Troyes aux XIV et $\mathrm{XV}^{\mathrm{e}}$ siècles », Mémoires de la société académique d'agriculture, des sciences, arts et belles lettres du département de l'Aube, 1964, vol. CIII, p. 5-17.

33 À Reims, par exemple: Julien Briand, «Foi, politique et information en Champagne au XV $\mathrm{XV}^{\mathrm{e}}$ siècle », Revue historique, vol. 653, nº 1, 2010, p. 59-97.
} 
soient deboutez de ceste ville que ledit frere Lyonnart ${ }^{34}$.

L'évêque de la ville, Jean Léguisé, connu pour ses accointances armagnaques dès son arrivée sur le siège épiscopal de Troyes en 1426, semble également être un des acteurs-clés de cette incitation à la fidélité, doublée d'une répression à l'endroit des traîtres ${ }^{35}$. Il est poussé à encourager la délation auprès de ses fidèles :

monseigneur le doien exposera et dira a monseigneur l'evesque qu'il lui plaise remonstrer demain, en sa predication au peuple, les grans perils qui par trahison se peuvent ensuir, et que il commende et ordonne a tous, a peine d'excommeniement, que se il est aucun qui saiche aucuns mauvaiz traitres aimant le party contraire du roy, que ilz lui facent savoir en confession ou autrement, afin que a l'aide de Dieu et par le moyen des bonnes creatures, la cité puisse estre preservé de traïson ${ }^{36}$.

Ici, l'excommunication est appelée en renfort pour favoriser la fidélité royale. À l'exclusion de la communauté urbaine s'ajoute la menace de l'exclusion de la communauté des croyants.

L'évêque joue aussi un rôle de diffusion des informations politiques, rôle-clé en période de conflit, et le conseil se doit de contrôler ses prédications. Lors de l'annonce de la mort du gouverneur de Champagne, le seigneur de Barbazan, grand fidèle du roi, le conseil a manifestement peur que cela n'émeuve le peuple et met au point une stratégie de communication circonstanciée, insistant sur le fait qu'il «n'a pas esté mort en la guerre ne pour le fait du roy, mais a esté mort en

\footnotetext{
${ }^{34}$ Troyes, AM, fonds Boutiot, série A, registre $1, \mathrm{f}^{\mathrm{o}} 129 \mathrm{v}^{\mathrm{o}}$.

${ }^{35}$ Sur le rôle important de l'évêque à Troyes : Françoise Bibolet, La Participation $d u$ clergé aux affaires municipales de la ville de Troyes aux XIV et $X V^{e}$ siècles, Troyes, Impr. Paton, 1946 ; et sur Jean Léguisé : Arthur Émile Prévost, Le Diocèse de Troyes. Histoire et documents, Dijon, Impr. de l'Union typographique, 1924, 3 vol, t. II, p. 33. ${ }^{36}$ Troyes, AM, fonds Boutiot, série A, registre $1, \mathrm{f}^{\circ} 46 \mathrm{r}^{\circ}$.
} 
Lorraine et pour la querelle de monseigneur le duc de Bar $^{37} \gg$. Il est précisé dans le registre que plus de 2000 personnes assistent à la nouvelle.

Enfin, les nombreux courriers reçus et envoyés par la ville participent de cette construction. Souvent, les autorités municipales ordonnent la lecture des lettres royales par un crieur en ville ${ }^{38}$. On les lit aussi lors des assemblées générales, où jusqu'à 300 personnes sont parfois rassemblées. En général, c'est l'occasion de donner des nouvelles du roi et de sa famille, de leur bonne santé, mais aussi de réaffirmer le fait que si les ennemis arrivaient, « le roy les secoureroit en sa personne ${ }^{39} »$.

C'est un conseil occupé à promouvoir la fidélité au roi qui est à l'œuvre, auprès de Troyens peut-être moins concernés.

\section{Fidélité royale et communauté : la construction d'une fiction?}

L'identification des dirigeants municipaux présents avant et après le retour à la fidélité du dauphin en 1429 révèle un renouvellement très faible. La comparaison des comptes des aides est ainsi intéressante puisque les principaux responsables ne se sont pas contentés d'administrer la ville en interne mais ont levé et prêté des sommes d'argent importantes à destination d'abord du duc de Bourgogne et du régent, puis du futur Charles VII. Sur 64 noms de personnes avec des responsabilités recueillis dans les recueils d'imposition, on retrouve 11 de

\footnotetext{
${ }^{37}$ Troyes, AM, fonds Boutiot, série A, registre $1, \mathrm{f}^{\mathrm{o}} 48 \mathrm{v}^{\mathrm{o}}$. « Item a esté deliberé que monseigneur l'evesque fera une collacion apres disner en remonstrant au peuple le cas advenu de la mort de monseigneur de Barbazan, et que esbau ne s'en doit car il y a petite perte, excepté de la personne de mondit seigneur de Barbazan [...] Item qu'il n'a pas esté mort en la guerre ne pour le fait du roy, mais a esté mort en Lorraine et pour la querelle de monseigneur le duc de Bar; [que] nous sommes bien fortiffiez et advitaillez [et que] nous sommes es mains de nostre souverain seigneur le Roy ».

${ }^{38}$ Troyes, AM, fonds Boutiot, série A, registre $1, \mathrm{f}^{\circ} 68 \mathrm{r}^{\circ} .:$ : Lesquelles lettres furent a tres grant reverance receues, ouvertes et leues en la presence d'iceulx messires les ambassadeurs et les habitans d'icelles villes comparans comme dessus. »

${ }^{39}$ Troyes, AM, fonds Boutiot, série A, registre $1, \mathrm{f}^{\mathrm{0}} 50 \mathrm{v}^{\mathrm{o}}$.
} 
ces personnes élues au conseil municipal et 35 présentes à ce même conseil $^{40}$. Ainsi, dans la majorité des cas, les responsables restent les mêmes, y compris ceux qui étaient proches des officiers du duc de Bourgogne. Si le bailli change, Jehan de Dinteville laissant la place à Antoine de Chabannes pour un mois en juillet 1429, puis à Guillaume Beslier, nommé par le roi le 29 juillet $1429^{41}$, ce n'est pas le cas de son lieutenant, Pierre le Tartrier, pourtant omniprésent dans les affaires bourguignonnes $^{42}$. On peut toutefois noter l'arrivée de Laurent Tourier, nouveau procureur des habitants, qui occupe une place importante dans le registre et qui est sans doute un des artisans de l'affirmation de la fidélité au roi par la ville. De même, on ne constate pas de nouvelle forme politique après l'arrivée du roi. Ce fait est encore plus frappant si l'on compare un feuillet de délibération de 1425 , seul à être conservé avant le registre. Sur les 30 noms des participants au conseil, 28 sont encore présents au conseil dans notre registre et la forme de la délibération reste la même ${ }^{43}$.

Que peut-on voir alors de l'opinion des habitants et de la communauté dans le registre? La rumeur et le débat sont évoqués au détour de certaines décisions, et les habitants rejoignent parfois les conseillers pour s'opposer à des mesures royales transmises par le bailli ou son lieutenant. Des résistances se forment, à la manière de ces collecteurs d'impôts qui refusent de rendre leurs comptes à la ville au début de l'année 1430 ou encore de contribuables refusant de payer ce

\footnotetext{
${ }^{40}$ Troyes, AM, fonds Boutiot, série F, registres 43, 44 et 45.

${ }^{41}$ Gustave Dupont-Ferrier, Gallia regia, op. cit., p. 66, no 22 524. Guillaume Beslier a ainsi le parcours exemplaire d'un fidèle du dauphin. Ce changement des officiers royaux avec les événements politiques s'observe tout aussi bien lors de la prise de la ville par les Bourguignons en 1417-1418, ainsi que l'explique Léonard Dauphant, Le Royaume des quatre rivières. L'espace politique français, 1380-1515, Seyssel, Champ Vallon, 2012, p. 309.

${ }^{42}$ Ibid., p. 74, n ${ }^{\mathrm{o}} 22544$.

${ }^{43}$ Troyes, AM, fonds Boutiot, AA7, liasse 1, pièce 1.
} 
même impôt ${ }^{44}$. Dans les comptes des deniers communs sont aussi gardées les traces de manouvriers ayant refusé de travailler ${ }^{45}$. À la fin de cette même année, un complot aurait été découvert, visant à faire revenir les Bourguignons, complot dans lequel des membres du conseil de ville sont incriminés ${ }^{46}$. Le 30 juin 1431, alors que le duc de Bourgogne est non loin de la ville, les opposants se font plus bruyants et les autorités redoublent d'attention :

Et c'est assavoir que l'en repute comme
souspeconnez tous ceulx qui sont trouvez
coupables de la traïson et qui d'icelle sont
accusez par un seul tesmoing, et aussi ceulx qui
ont eu leurs peres, leurs freres, leurs cousins
executez, et qui ont descousues leurs croix
comme par desrision en eulx resjoissant de ce
que l'en disoit des Borguignons. Item leur sera
deffendu qu'ilz ne communiquent ensemble, ne
facent assemblees, a peine de la hart ${ }^{47}$.

La haute main des officiers royaux sur la politique urbaine s'explique alors par la nécessité de réprimer ces oppositions, comme se souvient le procureur du roi une cinquantaine d'années plus tard, seule voix discordante parmi les récits sur l'obéissance et la loyauté sans faille des Troyens :

Et que [Troyes] a tousjours esté regie et gouvernee de son auctorité et par ses gens et officiers $[\ldots]$ et quant aucuns particuliers

\footnotetext{
${ }^{44}$ Troyes, AM, fonds Boutiot, série F, registre $45, \mathrm{f}^{\mathrm{o}} 8$ : les collecteurs d'impôt payent 16 sous 8 deniers tournois pour « informacion de pluseurs taverniers et autres gens qui vinrent acompaignez de pluseurs de leurs varlez a la sale et publierent ilec et autre part que on ne payast pour ledit impost et autres choses ».

${ }^{45}$ Troyes, AM, fonds Boutiot, série $\mathrm{B}$, registre $11, \mathrm{f}^{\mathrm{o}} 16 \mathrm{v}^{\circ}$ : amende pour «certain reffuz et deffaulx par luy faiz a aller aux corvuees qui se sont faictes en ceste annee, a amener matiers pour employer en la fortifficacion et emparement de ladite ville ». Nous n'avons pas de trace de situations comme celle-ci dans les comptes des autres années.

${ }^{46}$ Françoise Bibolet, «Les derniers fidèles au traité de Troyes en 1430 », La Vie en Champagne, $\mathrm{n}^{\circ} 44$, octobre-décembre 2005, p. 17.

${ }^{47}$ Troyes, AM, fonds Boutiot, série A, registre $1, \mathrm{f}^{\mathrm{0}} 45$.
} 
habitans se sont le temps passé efforcez d'eulx desloïaulter envers le roy leur souverain et naturel seigneur, lesdicts gens et officiers dudit sire y ont pourveu et remedié et en ont fait de grandes pugnicions qui sont notoires a chascun $^{48}$.

Enfin, sans parler d'opposition générale à la politique du dauphin, il apparaît que la communauté est le plus souvent dans une position de négociation et de pragmatisme qui tranche avec l'idée d'une fidélité politique retrouvée. Les intérêts et le discours de la ville restent les mêmes avant et après 1429, principalement la volonté de ne pas avoir de garnisons militaires dans ses murs et de garder une monnaie stable. En juillet 1432, le roi demande des vivres à la ville mais celle-ci retarde de plusieurs mois la réponse ${ }^{49}$. En octobre 1432, le conseil de ville refuse la décision royale de déprécier la monnaie ${ }^{50}$. Le rapport de force est permanent.

Finalement, ce sur quoi ce registre nous renseigne peut-être le plus, c'est sur la construction a posteriori de ce moment comme événement fondateur pour la communauté. Si le bailli encourage la fidélité au dauphin et poursuit les traîtres grâce à son contrôle sur les assemblées de la ville, les autres autorités municipales, les pratiques de gouvernement et les préoccupations quotidiennes restent les mêmes avant et après 1429. Et c'est sans doute justement ce caractère exogène de la fidélité troyenne qui incite Charles VII à mettre un temps le conseil de ville sous contrôle du bailli, avant que ce dernier ne délaisse progressivement la présidence des

\footnotetext{
48 Troyes, AM, fonds Boutiot, AA1, liasse 3, pièce 7, 8 avril 1483: Mémoire $d u$ procureur du roi contre les Troyens sur la question de l'échevinage.

${ }^{49}$ Troyes, AM, fonds Boutiot, série A, registre 1, $\mathrm{f}^{\circ}$ 94, 12 juillet 1432.

${ }^{50}$ Troyes, AM, fonds Boutiot, série $A$, registre $1, \mathrm{f}^{\mathrm{O}} 63$, en octobre 1432 : après avoir lu une lettre de Charles VII sur les monnaies, recopiée dans le registre, et délibéré du danger qu'elle représente pour la ville, la conclusion tombe : « et pour ce a esté advisé que publier lesdictes lettres estoit le domaige du Roy, de ses subgez et de la chose publique, et estoit expedient de surceoir ladite publication, ce qui a esté. ».
} 
assemblées municipales et ne cède son «auctorité de justice» aux « esleuz ${ }^{51} »$ troyens. L'entrée de Charles VII ne s'est sans doute pas faite dans la ferveur générale et ce registre s'inscrit dans le processus de reprise en main des villes conquises par Charles VII.

Pourtant, dès la fin $\mathrm{du} \mathrm{XV}^{\mathrm{e}}$ siècle, on cherche à valoriser cet épisode, dans le contexte de la construction de légitimité du nouvel échevinage qui gouverne la ville. Il devient un moment obligé du discours porté par la ville devant le roi ou encore des cartulaires regroupant la mémoire de la ville. On met l'accent sur le fait que Troyes est la première ville au Nord de la Loire à avoir reconnu l'autorité de Charles VII et sur l'unanimité de la communauté dans cette reconnaissance. C'est sans doute à ce moment que ces cahiers hétérogènes, peut-être conservés dans des lieux différents (archives municipales, archives du bailliage) sont reliés en un registre unique, conservé précieusement. Celui-ci est alors révélateur de la volonté des acteurs urbains de se réapproprier cet épisode et d'affirmer une fidélité au roi sans faille. Cette fiction d'une communauté politique homogène se retrouve ensuite sous la plume des historiens de la ville ${ }^{52}$. Ainsi, pour peu que le lien politique soit prégnant pour caractériser la communauté troyenne de 1429, il n'en est pas moins essentiel à la construction d'une mémoire collective.

\footnotetext{
${ }^{51}$ Les élus de la ville apparaissant dans le registre à partir du $1^{\mathrm{er}}$ octobre 1432.

${ }^{52}$ Se reporter à l'introduction. 